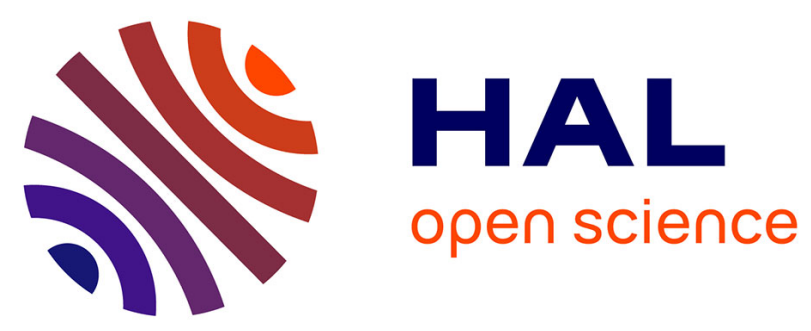

\title{
Parameters sensitivity analysis in charge transport model using Sobol indexes for optimization purpose
}

Fulbert Baudoin, Séverine Le Roy, G. Teyssedre, Christian Laurent, I. Alhossen, Florian Bugarin, Stéphane Segonds, Nicolas Binaud

\section{- To cite this version:}

Fulbert Baudoin, Séverine Le Roy, G. Teyssedre, Christian Laurent, I. Alhossen, et al.. Parameters sensitivity analysis in charge transport model using Sobol indexes for optimization purpose. IEEE International Conference on Dielectrics, 2016, Montpellier, France. 10.1109/ICD.2016.7547745 . hal02047977

\section{HAL Id: hal-02047977 \\ https://hal.science/hal-02047977}

Submitted on 2 Dec 2020

HAL is a multi-disciplinary open access archive for the deposit and dissemination of scientific research documents, whether they are published or not. The documents may come from teaching and research institutions in France or abroad, or from public or private research centers.
L'archive ouverte pluridisciplinaire HAL, est destinée au dépôt et à la diffusion de documents scientifiques de niveau recherche, publiés ou non, émanant des établissements d'enseignement et de recherche français ou étrangers, des laboratoires publics ou privés. 


\section{Parameters sensitivity analysis in charge transport model using Sobol indexes for optimization purpose}

\author{
F. Baudoin, S. Le Roy, G. Teyssedre, C. Laurent \\ Laplace, University of Toulouse, CNRS, INPT, UPS \\ Toulouse, France \\ fulbert.baudoin@laplace.univ-tlse.fr
}

\begin{abstract}
This paper aims at carrying out a parameters sensitivity analysis on a charge transport model using an approach based on the Sobol's method. Charge transport models generally encompass a large number of unknown or ill-defined parameters, typically 10 , that interact to produce macroscopic response observable through e.g. space charge density profiles and external current measurements. However, the various physical processes of the model have different impact on the predicted behaviour. The Sobol' approach applied herein is used to study how the variation of the current density and charges density can be quantitatively apportioned to the variation of the model input parameters.
\end{abstract}

Keywords: modelling - charge transport - Sobol indexes LDPE material - sensitivity analysis

\section{INTRODUCTION}

Various physical models have been implemented for describing the mechanisms of charge generation and transport in solid dielectrics [1-3]. These models require the handling of a number of parameters like injection barrier, mobility, trapping coefficient, etc. Most of these parameters cannot be determined by independent experiments and it is a heavy task to estimate parameter values that best fit experimental data. Optimization algorithms aim at systematizing this part of the modelling activity. However, to facilitate the convergence of optimization algorithms, it is important to quantify the effect of each input variable on the output observables in order to limit the optimization to the most influential variables.

The method implemented in this work, based on Sobol's analysis, guides the choice of optimization algorithms by focussing on the main parameters affecting the charge transport model which makes the resolution possible. The task is to build a sensitivity analysis on 4 of the inputs of the charge transport models regarding the impact on the space charge density and current measurements which are the main observables that can be implemented for feeding the models. With this in hands, the optimization can select the best conditions for appropriate estimation of model parameters.

\section{UNIPOLAR CHARGE TRANSPORT MODEL}

\section{A. Physical description and basic equations}

The model developed here is a unipolar description of charge transport based as detailed elsewhere [1]. This model considers two levels of charge traps: a deep trap level

\author{
I. Alhossen, F. Bugarin, S. Segonds, N. Binaud \\ University of Toulouse, UPS, INSA, ISAE \\ ICA, Toulouse, France \\ florian.bugarin@univ-tlse3.fr
}

accounting for relatively long-lasting trapping of charges and a shallow level to which is associated an effective mobility for mobile carriers. Charge carriers have a given probability to escape from deep traps by overcoming a potential barrier that is included in the de-trapping coefficients. Two kinds of species are considered, being mobile and trapped carriers. Even when neglecting dipolar processes and diffusion one generally has to solve the following coupled equations considering a 1D problem along the spatial coordinate $x$, whatever the model used to describe charge transport:

$$
\begin{gathered}
j(x, t)=\mu \cdot E(x, t) \cdot e \cdot n_{\mu}(x, t) \\
\frac{\partial n(x, t)}{\partial t}+\frac{1}{e} \frac{\partial j(x, t)}{\partial x}=s(x, t) \\
\frac{\partial E(x, t)}{\partial x}=\frac{e . n(x, t)}{\varepsilon}
\end{gathered}
$$

where $j$ is the transport current associated with mobile carriers of density $n_{\mu}$ and charge $e, \mu$ is the mobility, $E$ is the electric field, $n$ the total carriers density. The term $s$ is the source term, i.e. it encompasses any local charge density variation due to processes other than transport, such as the internal generation of charges. These equations may have a specific form for the interfaces, and are complemented by boundary conditions (e.g. applied electric field, etc.). An example of an expression for the source term of Eq. 2 is given for mobile carriers:

$$
s_{1}=\frac{d n_{\mu}}{d t}=-B n_{\mu}\left(1-\frac{n_{t}}{n_{0 t}}\right)+D n_{t}
$$

where $B$ is the trapping coefficient, $n_{\mu}$ and $n_{t}$ are respectively mobile and trapped carrier densities, $n_{0 t}$ is the maximal trap density, in our case $n_{0 t}$ is such that $e . n_{0 t}=100 \mathrm{C} . \mathrm{m}^{-3}$. The detrapping probability is defined by a de-trapping coefficient which is of the form:

$$
D_{e, h}=v \cdot \exp \left(\frac{-e w_{t r}}{k_{B} \cdot T}\right)
$$

where $v$ is the attempt to escape frequency, which has been set to $k_{B} T / h=6.210^{12} \mathrm{~s}^{-1}$ at room temperature, $T$ is the temperature and $w_{t r}$ is the de-trapping barrier. In this model, we have supposed that charge generation results from injection at the electrodes according to a corrected Schottky law (there is no injection when the electric field at the electrode is null): 


$$
j_{i n j}=A T^{2} \exp \left(-\frac{e w}{k_{B} T}\right)\left[\exp \left(\frac{e}{k_{B} T} \sqrt{\frac{e E_{\text {electrode }}}{4 \pi \varepsilon}}\right)-1\right]
$$

where $j_{i n j}$ is the flux of charge at the electrode, $k_{B}$ is the Boltzmann's constant, $\mathrm{A}=1.210^{6} \mathrm{~A} \cdot \mathrm{m}^{-2} \cdot \mathrm{K}^{-2}$ is the Richardson constant, $w$ is the injection barrier.

For this study, we consider low density polyethylene LDPE material, in film form of thickness $D=200 \mu \mathrm{m}$, at a temperature of $40^{\circ} \mathrm{C}$ and under a DC electric field of $30 \mathrm{kV} \cdot \mathrm{mm}^{-1}$ applied between the both electrodes during different times of polarization: $t_{p o l}$ of $100 \mathrm{~s}, 600 \mathrm{~s}$ and $1200 \mathrm{~s}$.

\section{SENSITIVITY ANALYSIS}

\section{A. Model as black box}

In this part, the model of charge transport described previously is viewed as a black box with some inputs and outputs that are defined as in Fig. 1.

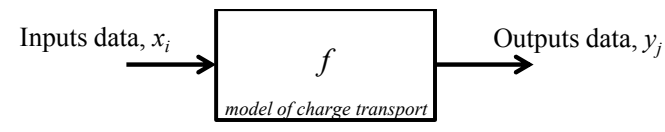

Fig. 1. Model as a black box

We denote input data some physical constants allowing describing the mechanisms of charge generation and transport in the solid insulation, sometimes the term parameters will be also used. In this work, only 4 inputs are considered and concern the charges injection, mobility of carriers and trapping and detrapping processes, Table I. We denote output data the main results obtained by the model. In our case, we consider only the net density of carriers and the current density because they are easily observable using experimental devices. The $f$ function corresponds to the charge transport model described previously and includes all the partial differential equations.

TABLE I. INPUT AND OUTPUT DATA

\begin{tabular}{lll}
\hline Inputs & Notation & Units \\
\hline Barrier height for injection, $w$ & $x_{1}$ & $\mathrm{eV}$ \\
Mobility, $\mu$ & $x_{2}$ & $\mathrm{~m}^{2} \cdot \mathrm{V}^{-1} \cdot \mathrm{s}^{-1}$ \\
Trapping coefficient, $B$ & $x_{3}$ & $\mathrm{~s}^{-1}$ \\
De-trapping barrier height, $w_{t r}$ & $x_{4}$ & $\mathrm{eV}$ \\
\hline Outputs & & \\
\hline Net carrier density & $y_{1}$ & $\mathrm{~m}^{-3}$ \\
Current density & $y_{2}$ & $\mathrm{~A} \cdot \mathrm{m}^{-2}$ \\
\hline
\end{tabular}

\section{B. Input boundaries}

The range set for the different inputs is shown in Table II. Lower and upper bounds are chosen first to be certain to keep physical sense to the conditions, second to have a large range of inputs in order to assume a broad and consistent representation of our output data, and lastly to have tractable computation. To the input range of each physical quantity, we suppose a renormalization can be achieved and after rescaling, the interval is supposed to be $[0,1]$. Indeed, it is useful for example to conceive each parameter as a random variable uniformly distributed over the $[0,1]$ interval, with all the input mutually independent.

TABLE II. INPUT RANGES

\begin{tabular}{lll}
\hline Inputs & Lower bound & Upper bound \\
\hline Barrier height for injection & $1.10 \mathrm{eV}$ & $1.20 \mathrm{eV}$ \\
Mobility & $10^{-14} \mathrm{~m}^{2} \cdot \mathrm{V}^{-1} \cdot \mathrm{s}^{-1}$ & $10^{-12} \mathrm{~m}^{2} \cdot \mathrm{V}^{-1} \cdot \mathrm{s}^{-1}$ \\
Trapping coefficient & $5 \times 10^{-4} \mathrm{~s}^{-1}$ & $10 \mathrm{~s}^{-1}$ \\
De-trapping barrier height & $0.73 \mathrm{eV}$ & $1 \mathrm{eV}$ \\
\hline
\end{tabular}

\section{The outputs as scalars}

In order to estimate the Sobol's indexes it is necessary to provide the outputs as scalars. Concerning the output $y_{1}$, which is normally a net carrier density profile, function of the position in the insulation and of the time, the scalar is obtained by integrating the net charge over the space and time as follows:

$$
y_{1}=\iint_{D t_{p o l}} n(x, t) \cdot d x \cdot d t
$$

For the current density, $y_{2}$, the output is obtained by:

$$
y_{2}=\int_{t_{p o l}} J_{t o t}(t) \cdot d t \text { with } J_{t o t}(t)=\frac{1}{D} \int_{D} j(x, t) \cdot d x
$$

\section{The principle of Sobol's indexes}

The goal of the parameters sensitivity analysis is to evaluate how the variation in an output can be apportioned to the variation of the inputs. In other worlds, the sensitivity parameter indicates how each input contributes to the output variability. This kind of tool could provide some important information before optimization processes to determine the influence of the parameters on the output of the charge transport model and so to classify parameters in order of influence. This leads to the determination of how the output is dependent on each of the inputs. Thus, sensitivity analysis allows the identification of the factor (or set of factors) that have the greatest influence on the response.

Usually sensitivity analysis methods are classified in two distinct groups: Local and Global. The local methods focus on the local outcome of the output by varying the inputs one at a time, while holding the others at some local values. Conversely, global sensitivity analysis methods examine the variation of the output while varying all the inputs over their entire ranges.

In this application, our focus is on the Sobol's method [4, 5], which is considered as one of the most powerful global sensitivity analysis methods. The principle of this method is to consider each input as varying randomly over its entire range. Then an output, as a function of the inputs, can be studied as a random variable, and thus its variability can be represented by the variance. The Sobol algorithm probes how the variance of the output can be decomposed into a sum of partial variances. Then, using this decomposition, it assigns to each input a sensitivity index representing the effect of this input on the output. So, if a general form of an input-output representation is written as:

$$
y_{k}=f\left(x_{1}, x_{2}, x_{3}, x_{4}\right)
$$


with $k=1$ for net density of charge or 2 for current density. The function $f$ represents the charge transport model.

Then, the sensitivity indexes are defined as:

$$
S_{i}=\frac{\operatorname{Var}\left(E\left[y_{k} \mid x_{i}\right]\right)}{\operatorname{Var}(y)}
$$

$S_{i}$ is called the first order Sobol's index of the input $x_{i}, E$ the mathematical expectation and $\operatorname{Var}$ the variance.

To clarify the concept behind Sobol's notation, let us consider the following. For predicting the influence of a certain input $x_{i}$ on the output $y_{k}$, one could fix this $x_{i}$ at some specific value $x^{*}$ and measure how $y_{k}$ varies while varying other inputs. If the variation of $y_{k}$ is small compared to that with all inputs varying, then $x_{i}$ does not have a real influence on $y_{k}$. However, if the variation of $y_{k}$ decreases, this means that $x_{i}$ is affecting $y_{k}$. Note that this variation of $y_{k}$ while fixing $x_{i}$ is quantified using $\operatorname{Var}\left(y_{k} \mid x_{i}=x^{*}\right)$. Now, we must consider that $x_{i}$ has different possible values over its entire range and is not just one specific $x^{*}$, thus it is required to take the mean of this conditional variance over the whole range of values of $x_{i}$. So the real reference for the sensitivity of $y_{k}$ with respect $x_{i}$ is $E\left[\operatorname{Var}\left(y_{k} \mid x_{i}\right)\right]$. Small values of $E\left[\operatorname{Var}\left(y_{k} \mid x_{i}\right)\right]$ indicate that $x_{i}$ actually affects $y_{k}$, large values indicate that $x_{i}$ has no strong effect on $y_{k}$. Knowing that:

$$
\operatorname{Var}(y)=E\left[\operatorname{Var}\left(y_{k} \mid x_{i}\right)\right]+\operatorname{Var}\left(E\left[y_{k} \mid x_{i}\right]\right)
$$

then $\operatorname{Var}\left(E\left[y_{k} \mid x_{i}\right]\right)$ can be also a reference for the sensitivity of $y_{k}$ with respect to $x_{i}$. Indeed, $\operatorname{Var}(y)$ is a constant positive quantity, then large values $\operatorname{Var}\left(E\left[y_{k} \mid x_{i}\right]\right)$ correspond to low values of $E\left[\operatorname{Var}\left(y_{k} \mid x_{i}\right)\right]$, and thus high sensitivity. However, small values of $\operatorname{Var}\left(E\left[y_{k} \mid x_{i}\right]\right)$ correspond to large values of $E\left[\operatorname{Var}\left(y_{k} \mid x_{i}\right)\right]$, and thus indicating less sensitivity. This interprets the notion of the Sobol's sensitivity indexes.

One can also calculate the index of order 2, for each pair of variables $\left(x_{m}, x_{n}\right)\left(x_{m}\right.$ is a variable different from $x_{n}$, chosen among the $p$ input variables, $p=4$ in our case) that represent the part of variance induced by the combination of variables $x_{m}$ and $x_{n}$. Therefore, for each variable $x_{m}$, there is $p$-1 order index 2. Similarly we can calculate indexes of higher order quantifying the part of total variance assignable to the combined variation of 3 or 4 variables. For each variable $x_{m}$, we can calculate the total sensitivity index corresponding to the sum of all orders sensitivity indexes involving the variable $x_{m}$. Note that the sum of the total sensitivity indexes of all the variables of the system is theoretically equal to one.

The different steps for Sobol sensitivity analysis:

1- Define the inputs $x_{i}$ and the lower and upper bound;

2- Generate the input sets over the defined ranges;

3- Run the input sets through the charge transport model;

4- Calculate the Sobol indices with Eq. (10);

5- Analyze the different order sensitivity indexes.

Note that, the Sobol's method, unlike many other sensitivity analysis methods, does not rely on any previous assumptions concerning the structure of the model under study. This is favorable in our case when studying the sensitivity of the logistic parameters, since we have no evidence about the form of the parameters in terms of $x_{i}$. In the next section, the Sobol's indexes of the factors $x_{i}$ associated with each of the outcomes $y_{k}$ will be displayed, this allows the ranking of factors according to their impact on the responses.

\section{RESULTS AND DISCUSSION}

\section{A. The various contributions to Sobol index}

The first result obtained concerns the influence of the inputs of the model on the net charge density. An electric field of $30 \mathrm{kV} / \mathrm{mm}$ is applied during $100 \mathrm{~s}$ on a LDPE material and the charge profiles are computed and integrated over time and space. Sobol index calculations are computationally expensive and require a high number of calls to the studied function. To illustrate this, Fig. 2 shows a result for the convergence as a function trial number. Two trials are made at each step for each input. It can be seen that about 50000 runs are necessary to reach convergence. This means 100000 calls to functions for each parameter, and in total 500000 calls for the all input parameters to guarantee the convergence of the Sobol's index. It represents about 30 hours to implement with a computer having an Intel Core i7-4790 processor, CPU of $3.60 \mathrm{GHz}$ and $32 \mathrm{Go}$ of RAM.

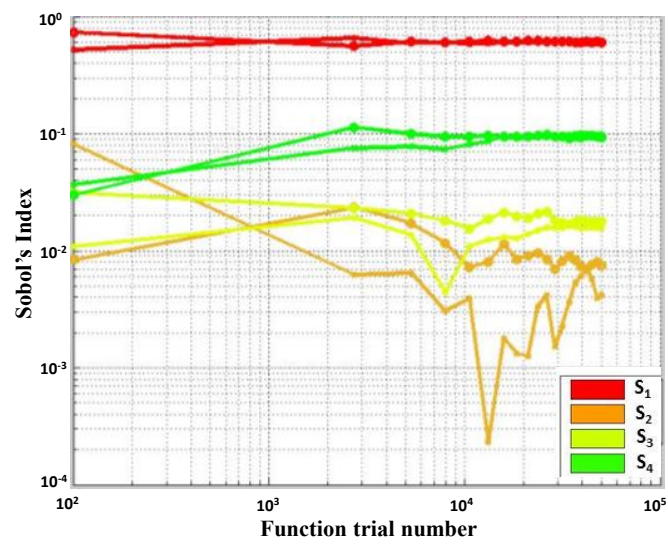

Fig. 2. Convervenge for the Sobol indexes calculation

Fig. 3 quantifies the amount of variance that each inputs $x_{i}$ contributes to the total variance of the net charge density and current density. We note $S_{i}$ the Sobol index of the $x_{i}$ input and $S_{i j}$ the index of order 2 between two inputs $x_{i}$ and $x_{j}$. As shown on this figure, the variance of a give contribution can be split in two parts: that caused by variations of individual inputs, which represent the main effect, and that caused by mutual interactions of several inputs. The sum of the total index reaches 1, which proves the independence of the different inputs and a good convergence of the algorithm (a value higher than 1 would mean redundancy between some input parameters). Concerning the Sobol's index for the net charge output, Fig. 3 shows that the barrier height of injection, $x_{1}$, plays an essential role in the first instants of the polarization: Sobol's index for the net charge density is affected by the charge injection at up to $60 \%$. The de-trapping barrier and the interaction between injection / de-trapping represent only $10 \%$ each to the total variance of the $y_{1}$ output. Other inputs are negligible, meaning that, at the first moments of the polarization, mobility and trapping do not have substantial influence on the net charge density. This first result shows that injection phenomena could be analyzed nearly independently of the other physical phenomena. For parameters optimization, 
the barrier height of injection could be estimated by fixing the others inputs at any value. Concerning the Sobol index for the current density output, the mutual interactions of inputs are more impacting that the individual inputs. The interaction injection/de-trapping represents $30 \%$ of the total variance while the individual input de-trapping concerns slightly less than $20 \%$ and injection phenomena about $10 \%$. Note here that the total sum of indexes is less than 1, meaning that higher order interactions (involving 3 input parameters) are at play.

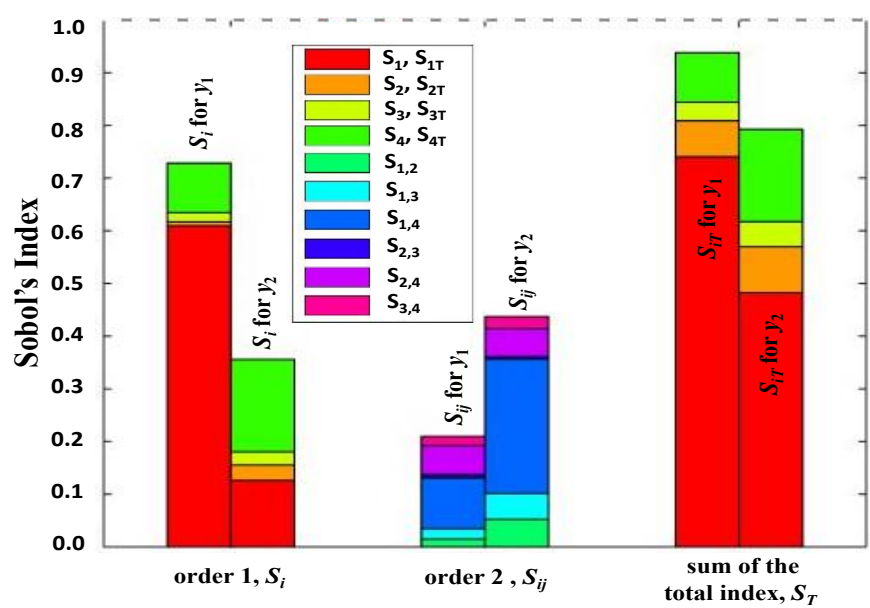

Fig. 3. Sobol indexes for the $y_{1}$ and $y_{2}$ output with charging time of $100 \mathrm{~s}$

\section{B. Impact of charging time}

Fig. 4a) shows the evolution of Sobol indexes during polarization process for the $y_{1}$ output (representing charge density) with charging times of $100 \mathrm{~s}, 600 \mathrm{~s}$ and $1200 \mathrm{~s}$. As explained previously, at the first moment of the polarization the injection process overwhelms the other parameters in terms of influence on the charge density. This trend tends to weaken over time. For $1200 \mathrm{~s}$, de-trapping rises up to $55 \%$ while barrier height of injection drops to less than $20 \%$. The other parameters, mobility and trapping do not have much influence on the output, at most $10 \%$ of the total variance. Finally, injection process, de-trapping and their interactions are impacting at up to $80 \%$. Concerning indexes for the $y_{2}$ output i.e. current density, there is not much evolution over time, cf. Fig 4b). Results show that interaction of order 2 is more impacting that the individual inputs. Here again, the sum of the total index does not reach 1 .

By analyzing these results a strategy of study can be designed for parameters optimization. The first step would consist in fixing mobility and trapping coefficient to a random value and then optimizing de-trapping coefficient and barrier height of injection with the net charge density only, preferably using short time data. By constraining the variation interval of estimated parameters found in the first step, the optimization could then be realized with the current density as output and the others parameters, trapping and mobility, as inputs. Indeed, interactions between parameters being more important with the $y_{2}$ output, the optimization process could adjust more precisely each one.

Beyond the current objectives, reverse uncertainty propagation can also be applied for identifying experimental favourable conditions to reduce uncertainties on the parameters. For instance, local sensitivity indexes can be evaluated for experimental conditions (temperature, field, etc..) allowing detecting the configuration that is the less dependent on the most uncertain parameters. The measurements coming from these specific configurations could be used to calibrate the models.

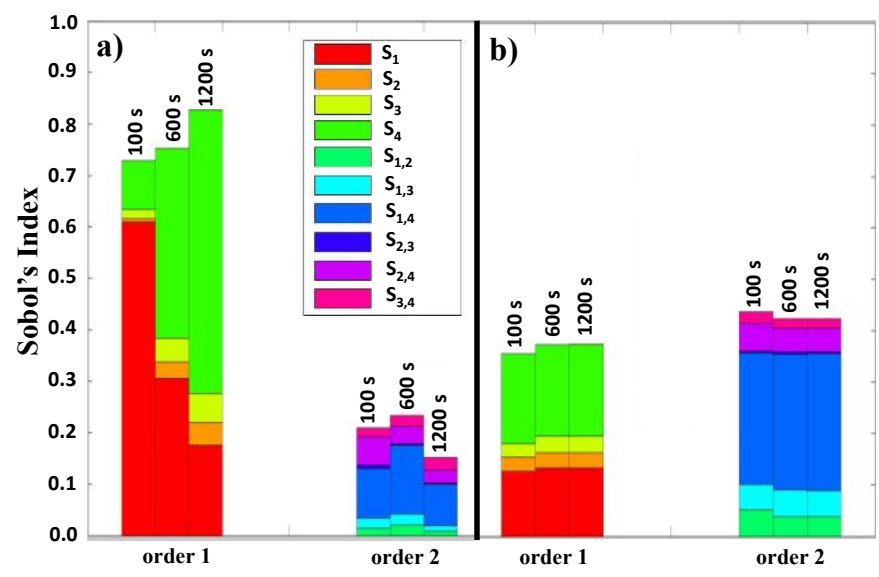

Fig. 4. Evolution of Sobol indexes during polarization process with charging times of $100 \mathrm{~s}, 600 \mathrm{~s}$ and $1200 \mathrm{~s}$ a) for the $y_{1}$ output and b) for the $y_{2}$ output

\section{CONCLUSION}

A Sobol algorithm has been used in order to estimate the impact of parameters of a charge transport model on the observables for the charge build up in insulation that are charge density measurements and external current. The procedure has been tested on 4 variable parameters as model input, considering charge density and current predicted for up to 20 min charging time. We show that at short charging time (100s), the main influent model parameters for the charge distribution are barrier to injection and de-trapping coefficient. The exact reason for the impact of de-trapping is not really elucidated at present. For longer polarization time, the contribution from detrapping rises and that from injection drops. Regarding charging current, the contributions to the Sobol index are mostly from mutual interactions between input parameters: this means that current analysis alone cannot easily unravel the different contributing processes to the macroscopic behaviour.

\section{REFERENCES}

[1] S. Le Roy, G. Teyssedre, G.C. Montanari, F. Palmieri, and C Laurent, "Description of charge transport in polyethylene using a fluid model with a constant mobility: fitting model and experiments", J. Phys. D: Appl. Phys., vol. 39, pp. 1427-1436, 2006.

[2] J. M. Alison and R. M. Hill, "A model for bipolar charge transport, trapping and recombination in degassed crosslinked polyethylene", $J$. Phys. D: Appl. Phys., vol. 27, pp. 1291-1299, 1994.

[3] K. Kaneko, T. Mizutani and Y. Suzuoki, "Computer simulation on formation of space charge packets in XLPE films", IEEE Trans. Dielectr. Electr. Insul., vol. 6, pp. 152-158, 1999.

[4] S. Segonds, C. Gogu, Y. Qiu, C. Bes, A. Mauffrey. "Design optimization under evidence based uncertainty with application to an engine connecting rod", International Journal of Design and Innovation Research, 5 (3), pp. 77-91, 2010.

[5] I.M. Sobol. "Global sensitivity indices for nonlinear mathematical models and their Monte Carlo estimates", Mathematics and Computers in Simulation (55), pp. 271-280, 2001 